\title{
Un enfoque general de la investigación de mercado
}

\author{
A general approach to market research \\ Miriam de la Cruz Ontiveros $^{a}$, Nayeli Melena Torres ${ }^{b}$, Jaime Garnica González ${ }^{c}$, Heriberto \\ Nicolas Morales ${ }^{d}$, Eva Selene Hernández Gress ${ }^{e}$
}

\begin{abstract}
:
A market research tries to minimize the commercial risks of a product or a service but is not a guarantee of success in its commercialization. It must be organized in two phases. The first phase is the planning where the problem, the theoretical framework, the study design and the activity schedule are formulated. The second phase is made up by the sample preparation, the data collection and its process, the analysis results and its making and finally the report. The research can be basic or applied. Could be in a qualitative way where one considers the knowledge of a marketing situation related to nature. Could be in a quantitative way where the measurement settings of a certain population give us a result. While the focus of the product research, can be either for final consumption or average consumption. The market research approach depends on the variables of the survey and the technique procedures to obtain information from the primary and secondary sources. It should be mentioned that all collected data must have a utility that does not influence in the decision making.
\end{abstract}

Keywords:

General approach, market research

\section{Resumen:}

Con una investigación de mercados, se busca minimizar los riesgos comerciales de un producto o servicio, pero no es una garantía de éxito en su comercialización. Por lo que se debe de organizar de dos etapas. La primera es la planeación donde se formula el problema, el marco teórico, diseño del estudio y programación de actividades. La segunda está integrada por la preparación de la muestra, recolección de datos y su procesamiento, análisis de resultados y la elaboración y presentación del informe. La investigación puede ser básica o aplicada, de corte cualitativa donde se profundiza en el conocimiento de una situación de marketing en conceptos y manifestaciones de la naturaleza. Cuantitativa orientada a la medición de parámetros de una población para llegar a un resultado concluyente. Mientras que el enfoque de la investigación del producto, puede ser de consumo final o consumo intermedio. El planteamiento de la investigación de mercados depende de las variables en estudio y los procedimientos de las técnicas a utilizar para la obtención de datos tanto en fuentes primarias como secundarias. Cabe hacer mención que cada dato a recolectar debe tener una utilidad de no influir significativamente en la toma de decisiones.

\section{Palabras Clave:}

Enfoque general, investigación de mercados

\section{Introducción}

\footnotetext{
${ }^{a}$ Universidad Autónoma del Estado de Hidalgo, Instituto de Ciencias Básicas e Ingeniería, Ingeniería Industrial, Email: mdlcontiveros@gmail.com

${ }^{\mathrm{b}}$ Universidad Autónoma del Estado de Hidalgo, Instituto de Ciencias Básicas e Ingeniería, Ingeniería Industrial, Email: nayeli_industrial@hotmail.com

c Autor de correspondencia, Universidad Autónoma del Estado de Hidalgo, Instituto de Ciencias Básicas e Ingeniería, Área Académica de Ingeniería y Arquitectura, Email: jgarnica@uaeh.edu.mx

d Universidad Autónoma del Estado de Hidalgo, Instituto de Ciencias Básicas e Ingeniería, Área Académica de Ingeniería y Arquitectura, Email: hnicolas@uaeh.edu.mx

e Universidad Autónoma del Estado de Hidalgo, Instituto de Ciencias Básicas e Ingeniería, Área Académica de Ingeniería y Arquitectura, Email: evah@uaeh.edu.mx
} 
El Mapa Mental, tiene la finalidad de visualizar el proceso, las fuentes de investigación, tipos de investigación y principales aplicaciones de un enfoque general de mercados. Para tal fin, se construyó la figura 1 con base en Orozco (1999) y Malhotra (2008).

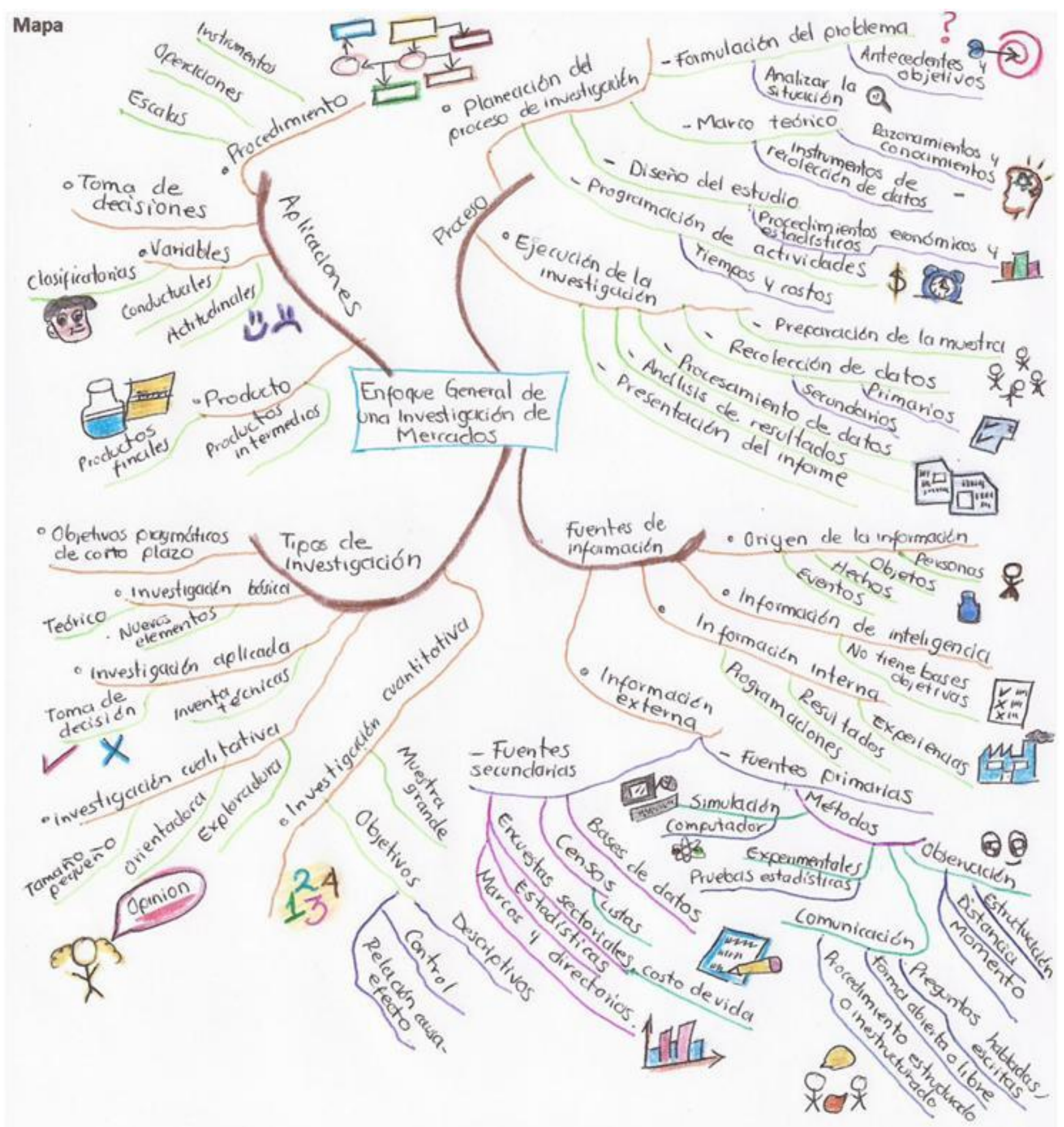

Figura 1. Mapa mental de un enfoque general de la investigación de mercado 


\section{Referencias}

[1] Malhotra, Naresh K. (2008). Investigación de mercados. México: Prentice Hall Ediciones.

[2] Orozco J., Arturo. (1999). Investigación de mercados conceptos y prácticas. Colombia: Editorial Norma. 